\title{
Efficacy of Molecular Response at 1 or 3 Months after the Initiation of Dasatinib Treatment Can Predict an Improved Response to Dasatinib in Imatinib-Resistant or Imatinib- Intolerant Japanese Patients with Chronic Myelogenous Leukemia during the Chronic Phase
}

\author{
Koiti Inokuchi, ${ }^{1)}$ Takashi Kumagai, ${ }^{2)}$ Eri Matsuki, ${ }^{3)}$ Kazuteru Ohashi, ${ }^{4}$ Atsushi Shinagawa, ${ }^{5)}$ \\ Yoshihiro Hatta, ${ }^{,}$Jin Takeuchi, ${ }^{6}$ Chikashi Yoshida, ${ }^{7}$ Hisashi Wakita, ${ }^{8}$ Yasuji Kozai, ${ }^{9}$ \\ Yukari Shirasugi, ${ }^{10}$ Shin Fujisawa, ${ }^{11)}$ Osamu Iwase, ${ }^{12)}$ Shingo Yano, ${ }^{13)}$ Shinichiro Okamoto, ${ }^{3)}$ \\ Koji Oba ${ }^{14)}$ Junichi Sakamoto, ${ }^{15)}$ and Hisashi Sakamaki ${ }^{4)}$
}

\begin{abstract}
Dasatinib is a BCR-ABL kinase inhibitor with improved potency compared with imatinib, for which efficacy and safety in imatinib-resistant and imatinib-intolerant patients with chronic myelogenous leukemia (CML) have been established. Here, an open-label phase II study evaluated the efficacy and safety of dasatinib in 50 Japanese patients with imatinib-resistant or imatinib-intolerant CML during the chronic phase (CML-CP). Dasatinib was effective in imatinib-resistant and imatinibintolerant patients. After 12 months of dasatinib therapy, 35 patients (70\%) had achieved a major molecular response (MMR) and 16 patients (32\%) had achieved a complete molecular response (CMR). Among the imatinib-resistant CML-CP cohort, 21 and 8 patients had achieved an MMR and a CMR after 12 months of dasatinib therapy, respectively. Among the imatinibintolerant CML-CP cohort, 14 and 8 patients had achieved an MMR and a CMR after 12 months of dasatinib therapy, respectively. After 18 months of dasatinib therapy, 38 out of 50 patients (76.0\%) had achieved an MMR and 19 patients (38. $0 \%$ ) had achieved a CMR. A lower level of $B C R-A B L$ transcript at 1 or 3 months after the initiation of dasatinib treatment was more strongly correlated with the $B C R-A B L$ transcript level at 12 and 18 months $(p<0.001)$ than a higher level of $B C R-A B L$. The T315I mutation was identified in two patients receiving dasatinib therapy. Dasatinib was generally well tolerated, with only 3 patients (5\%) having treatment discontinuation as a result of adverse hematologic events (thrombocytopenia, anemia, neutropenia) and/or non-hematologic events at a 12-month follow-up evaluation. Dasatinib was a safe and effective treatment for Japanese patients with imatinib-resistant or imatinib-intolerant CML. In addition, the molecular response at 1 or 3 months predicted a response to dasatinib at 12 or 18 months. [J Clin Exp Hematop 54(3) : 197-204, 2014]
\end{abstract}

Received: July 23, 2014

Revised : August 8, 2014

Accepted: September 4, 2014

${ }^{1)}$ Division of Hematology, Department of Internal Medicine, Nippon Medical School, Tokyo, Japan

${ }^{22}$ Department of Hematology, Ohme Municipal General Hospital, Tokyo, Japan

${ }^{3}$ Division of Hematology, Department of Internal Medicine, Keio University School of Medicine, Tokyo, Japan

${ }^{4}$ Hematology Division, Tokyo Metropolitan Cancer and Infectious Diseases Center, Komagome Hospital, Tokyo, Japan

${ }^{5}$ Department of Internal Medicine, Hitachi General Hospital, Hitachi, Ibaraki, Japan

${ }^{6}$ Department of Hematology and Rheumatology, Nihon University School of Medicine, Tokyo, Japan

"Department of Hematology, National Hospital Organization Mito Medical Center, Ibaraki, Japan

${ }^{8)}$ Division of Hematology and Oncology, Japanese Red Cross Society, Narita Red Cross Hospital, Narita, Japan

\footnotetext{
${ }^{9}$ Department of Hematology, Tokyo Metropolitan Tama Medical Center, Tokyo, Japan ${ }^{100}$ Division of Hematology, Department of Internal Medicine, Tokai University School of Medicine, Isehara, Japan

"Department of Hematology, Yokohama City University Medical Center, Yokohama, Japan

${ }^{122}$ Division of Hematology, Hachioji Medical Center, Tokyo Medical University, Tokyo, Japan

${ }^{13}$ Division of Clinical Oncology and Hematology, Department of Internal Medicine, Jikei University School of Medicine, Tokyo, Japan

${ }^{14}$ Interfaculty Initiative in Information Studies, The University of Tokyo, Tokyo, Japan \& Department of Biostatistics, School of Public Health, Graduate School of Medicine, The University of Tokyo, Tokyo, Japan

${ }^{15}$ Tokai Central Hospital, Kakamigahara, Japan

Corresponding author: Professor Koiti Inokuchi, Department of Hematology, Nippon Medical School, 1-1-5 Sendagi, Bunkyo-ku, Tokyo 113-8603, Japan

E-mail: inokuchi@nms.ac.jp
} 
Keywords: CML, dasatinib, imatinib-resistant, imatinib-intolerant, BCR/ABL

\section{INTRODUCTION}

Chronic myeloid leukemia (CML) is caused by abnormalities in hematopoietic stem cells resulting in the uncontrolled proliferation of cells originating from the bone marrow. The BCR-ABL fusion protein produced by the Philadelphia chromosome $(\mathrm{Ph})$ is a major molecular cause of $\mathrm{CML}{ }^{1}$

Imatinib is a selective BCR-ABL inhibitor that is effective against CML. ${ }^{2-4}$ However, resistance to imatinib gradually develops in many patients with CML who are treated with imatinib, and $31 \%$ of these patients discontinue imatinib treatment within 5 years because of insufficient responses or unacceptable toxicity. ${ }^{5}$ As major factors responsible for the development of resistance to imatinib, numerous point mutations in $B C R-A B L$ have been reported. ${ }^{6-8}$ Additional factors including BCR-ABL gene amplification, ${ }^{6,9}$ excretion of the drug through a P-glycoprotein efflux pump,,${ }^{10,11}$ and the activation of a signal transduction pathway for SRC family kinase and other signals ${ }^{12,13}$ have also been implicated in the development of resistance. In this regard, the development of new treatments is needed for patients with insufficient responses to imatinib and in whom imatinib cannot be continued at an effective dose because of toxicity.

Dasatinib (BMS-354825) is an oral tyrosine kinase inhibitor that exerts inhibitory activity against BCR-ABL and SRC family kinase. It binds in vitro to both active and inactive $\mathrm{BCR}-\mathrm{ABL}$ and is 325 times more potent than imatinib and 16 times more potent than nilotinib against wild-type BCR-ABLexpressing cells. ${ }^{14}$ Five phase 2 studies, collectively known as START (SRC/ABL Tyrosine kinase inhibition Activity Research Trials of dasatinib), have demonstrated that dasatinib is safe and can elicit a hematologic and cytogenetic response in patients with imatinib-resistant or imatinibintolerant CML or Ph-positive acute lymphocytic leukemia. ${ }^{15-18}$ Dasatinib was shown to be highly effective, with $91 \%$ of patients in the chronic phase of CML (CML$\mathrm{CP}$ ) exhibiting a complete hematologic response and $62 \%$ exhibiting a major cytogenetic response. The efficacy of dasatinib for CML-CP was durable, and the rate of a major cytogenetic response was $88 \%$; the progression-free survival rate was $80 \%$ and the overall survival rate was $94 \%$ at a 2 year follow-up. ${ }^{19}$ However, clinical data for second-line dasatinib therapy are lacking for imatinib-resistant and imatinibintolerant CML-CP in Japan.

In the present study, we conducted an open-label phase 2 study of dasatinib in Japanese patients with imatinib-resistant or imatinib-intolerant CML-CP. To determine the molecular responses to dasatinib, $B C R-A B L$ transcripts in the peripheral blood were evaluated using quantitative reverse transcriptasepolymerase chain reaction (Q-RT-PCR) at baseline and every month during the study; the results were then compared with the levels of total $A B L$ transcripts.

\section{PATIENTS AND METHODS}

\section{Patients and treatment}

This study was a phase II analysis of a clinical trial conducted by the Kanto CML Study Group (registered at http:// clinicaltrials. gov as NCT00866736). The study was conducted in accordance with the Declaration of Helsinki. The protocol was reviewed and approved by a recognized ethics review committee in each institution that participated in this study. Informed consent was obtained from all patients before entry into this study.

Japanese patients with CML-CP who were resistant or intolerant to first-line imatinib therapy participated in a prospective phase II study in order to evaluate the efficacy and safety of second-line dasatinib therapy. Patients who were at least 15 years of age were eligible for entry if they had imatinib-resistant or imatinib-intolerant CML-CP. The chronic phase of CML was defined as less than $15 \%$ blasts in the peripheral blood and bone marrow, less than $20 \%$ basophils in the peripheral blood, less than $30 \%$ blasts plus promyelocytes in the peripheral blood and bone marrow, a platelet count of at least $100 \times 10^{9} / \mathrm{L}$ unless thrombocytopenia was caused by recent therapy, and no extramedullary involvement other than in the liver or spleen. Patients with prior accelerated-phase or blast crisis CML were not eligible for inclusion in the trial.

Imatinib resistance was defined as the lack of a partial cytogenetic response after 3 months of imatinib treatment, the lack of a complete cytogenetic response (CCyR, $0.1-1.0 \%$ of $B C R-A B L / A B L)$ after 6 months of treatment, or the lack of a major molecular response (MMR, less than $0.1 \%$ of $B C R$ $A B L / A B L)$ after 12 months of treatment. Imatinib intolerance was defined as a non-hematologic toxicity of at least grade 2 or a hematologic toxicity of grade 3 or 4 persisting for more than 7 days in response to treatment with imatinib at a dose of $400 \mathrm{mg}$ or more.

Patients with $B C R-A B L$ mutations, which are associated with dasatinib resistance, ${ }^{14}$ were excluded from the analysis. None of the patients exhibited neutropenia $(<1,000 / \mu \mathrm{L})$ at the start of dasatinib treatment.

The real-time quantitative reverse transcriptionpolymerase chain reaction (RQ-PCR) analysis was performed by Bio Medical Laboratories (BML, Inc., Tokyo, Japan). Thus, the level of $B C R-A B L$ transcripts is shown using conversion factor (CF). ${ }^{20}$ Specifically, the level of measured $B C R-A B L$ transcripts was multiplied by $\mathrm{CF}$ and the level of international-scale $B C R-A B L$ transcripts is shown in this manuscript. ${ }^{20,21}$ A complete molecular response (CMR) was 


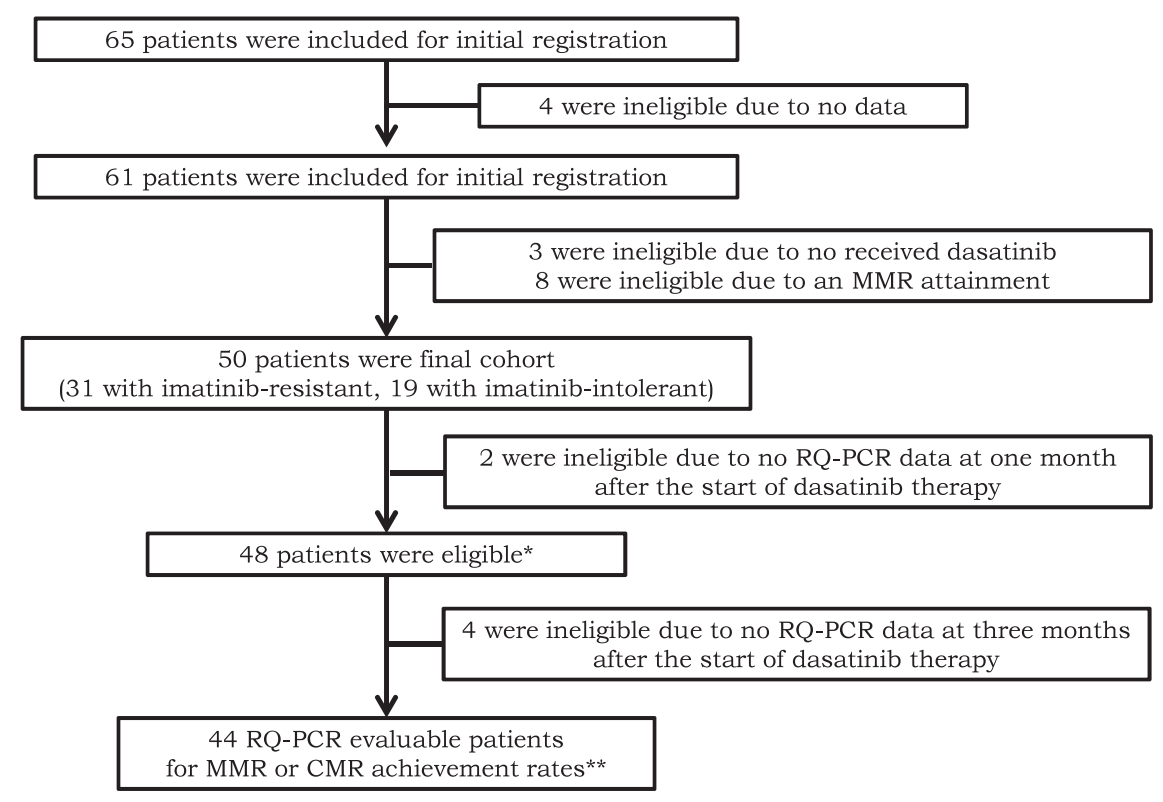

Fig. 1. Patient flow diagram. There was more than one reason for exclusion of patients. *, number of analyzed patients in Table $3 ; * *$, number of analyzed patients in Table 4; MMR, major molecular response; RQ-PCR, real-time quantitative reverse transcription-polymerase chain reaction; $\mathrm{CMR}$, complete molecular response

defined as a peripheral $B C R-A B L / A B L$ transcript ratio below the detection limit of the RQ-PCR analysis widely used throughout Japan, that is, a peripheral $B C R-A B L / A B L$ ratio < $10^{-4.16}(0.0069 \%)$ on the international scale (IS). ${ }^{20}$ MMR was defined as $0.1 \%$ of $B C R-A B L / A B L$.

\section{Treatment with dasatinib}

Patients received an oral dose of $100 \mathrm{mg}$ of dasatinib once daily. Therapy could be interrupted or reduced to $70 \mathrm{mg}$ daily or $50 \mathrm{mg}$ daily in response to a hematologic toxicity of at least grade 3 or a non-hematologic toxicity of at least grade 2. When therapy was interrupted, the treatment was then reinitiated at a reduced dose level or discontinued altogether, depending on the severity of the adverse event and on the number of times the same event had occurred. In addition, the investigator and the sponsor made all dose reduction or discontinuation decisions for patients with any signs of bleeding or hemorrhage of any grade. Follow-up for 18 months has been performed in all cases.

\section{Patient evaluations}

The cytogenetic and hematologic responses to dasatinib were monitored using peripheral blood samples. Complete blood counts were analyzed once weekly for the first 12 weeks and every 3 months thereafter. The assessment of drug toxicities was continuous and included a physical examination to monitor adverse events that was conducted weekly for the first month and every 4 weeks thereafter. mRNA was collected from the peripheral blood samples and was analyzed for $B C R-A B L$ gene point mutations using denaturing highperformance liquid chromatography and sequencing; the level of expression was examined using RQ-PCR. To determine the molecular responses to dasatinib, the $B C R-A B L$ transcripts in peripheral blood were evaluated using Q-RT-PCR at baseline, one month, and every three months during the study, and the results were compared with the levels of total $A B L$ transcripts. $^{20,21}$ Cytogenetic responses were based on the prevalence of Ph-positive interphase among at least 100 leukocytes in each peripheral blood sample. We used fluorescence in situ hybridization with BCR and ABL double-color probes to detect $\mathrm{Ph}$-positive leukocytes. ${ }^{22,23}$ The criteria for cytogenetic responses according to the percentage of $\mathrm{Ph}$ positive cells in interphase among the peripheral leukocytes were as follows: CCyR, $0 \%$; a partial cytogenetic response, $1 \%$ to $35 \%$; minor cytogenetic response, $36 \%$ to $65 \%$; minimal cytogenetic response, $66 \%$ to $95 \%$; and no cytogenetic response, $96 \%$ to $100 \%$.

\section{Statistical analysis}

The primary endpoint was MMR rate in CML patients at 12 months. Secondary endpoints were safety after treatment with dasatinib, CMR rate, and efficacy for patients with $B C R$ $A B L$ point mutations. On the basis of previous evidence, ${ }^{24}$ we 
Table 1. Patient characteristics

\begin{tabular}{|c|c|c|c|}
\hline Clinical feature & Total & Imatinib-resistant & Imatinib-intolerant \\
\hline Registration number & $61(50)^{*}$ & $36(31)^{*}$ & $25(19)^{*}$ \\
\hline Median age, year (range) & $57\left(16^{-91)}\right.$ & $57\left(16^{-74)}\right.$ & $57(24-91)$ \\
\hline Male & 41 & 24 & 17 \\
\hline Female & 20 & 12 & 8 \\
\hline
\end{tabular}

*, Data is for the final analyzed cohort.

set our goal at an MMR rate of $35 \%$. In this situation, the required sample size was 55 patients to detect a difference between a threshold MMR rate of $15 \%$ and a target MMR rate using exact test for single proportion with one-sided alpha error of $2.5 \%$ and statistical power of $90 \%$. To account for dropouts, the number of patients to be accrued was set at 60 in total.

The observed MMR rate at 12 months was compared with $15 \%$ using binomial test in the primary analysis. We also calculated the exact $95 \%$ confidence intervals (CI) for each proportion. The MMR rate and the CMR rate were estimated at each follow-up time. The associations between molecular response at 1 month or 3 months and the response at 12 months were evaluated using a chi-squared test. All analyses were performed using SAS version 9.3.

\section{RESULTS}

Sixty-five patients were enrolled between March 2009 and March 2010. Four of these 65 patients were determined to be ineligible for inclusion in the study, so they were excluded (Fig. 1). Three patients with imatinib-intolerant CML-CP ultimately did not receive dasatinib, so these patients were also excluded from the present analysis. Since eight of the registered patients had attained an MMR at the time of registration, these eight patients were additionally excluded. ${ }^{20}$ The remaining 50 patients were analyzed statistically. Table 1 shows the characteristics of the patients. The results presented are for the final cohort of 50 patients: 31 with imatinib-resistant CML-CP and 19 with imatinib-intolerant CML-CP.

The patient demographics and baseline disease characteristics are representative of the patient population with imatinib-resistant or imatinib-intolerant CML-CP (Table 1).

\section{Dosage of dasatinib}

All the patients received an oral dose of $100 \mathrm{mg}$ of dasatinib once daily at baseline. When a hematologic toxicity of at least grade 3 or a non-hematologic toxicity of at least grade 2 was observed, the dosage of dasatinib was reduced or transiently interrupted. Thus, dasatinib was continued at 100 $\mathrm{mg}$ /day for 12 months in $20(40 \%)$ of the 50 patients. The dose intensity at 12 months was $82.0 \mathrm{mg} /$ day. Six patients discontinued dasatinib treatment after 12 months. There were no patients who died in 12 months.

\section{Molecular response}

After 12 months of dasatinib therapy, 35 patients $(70 \%$; 95\% CI $=55.4 \%-82.1 \%)$ had achieved an MMR and 16 patients $(32 \%$; $95 \% \mathrm{CI}=19.5 \%-46.7 \%)$ had achieved a CMR. Among the imatinib-resistant CML-CP cohort, 21 and 8 patients achieved an MMR and a CMR, respectively. The observed MMR rate showed a statistically significant difference with a threshold MMR rate of $15 \%(p<0.0001)$. Among the imatinib-intolerant CML-CP cohort, 14 and 8 patients achieved an MMR and a CMR, respectively. All of the 50 patients were followed for 18 months. After 18 months of dasatinib therapy, 34 out of 50 patients $(68.0 \%$; $95 \% \mathrm{CI}=53.3$ to $80.5 \%$ ) had achieved an MMR and 22 patients $(44.0 \% ; 95 \% \mathrm{CI}=30.0$ to $58.8 \%)$ had achieved a CMR.

\section{Prediction of an improved response to dasatinib at 12 or 18 months based on the efficacy of the molecular re- sponse at 1 or 3 months after the initiation of dasatinib treatment (Fig. 2)}

To investigate the relationships between response and outcome, the 50 patients were divided into four groups according to the therapeutic effect of dasatinib (Table 2): Group A exhibited more than $10 \% B C R / A B L$ mRNA, Group B exhibited 1\%-10\% BCR/ABL mRNA, Group C exhibited 0 . $1 \%-1 \% B C R / A B L$ mRNA, and Group D exhibited less than 0 . $1 \% B C R / A B L$ mRNA. At baseline, Group A was composed of 16 patients, Group B was composed of 13 patients, Group C was composed of 21 patients, and Group D did not contain any patients. After one month of dasatinib treatment, Group A was composed of 9 patients, Group B was composed of 10 patients, Group C was composed of 18 patients, and Group D was composed of 11 patients (Table 3). Three months after dasatinib treatment, Group A was composed of 4 patients, Group B was composed of 4 patients, Group C was composed of 15 patients, and Group D was composed of 21 patients (Table 4). Figure 2 shows the rates of MMR and CMR achievement at both 12 and 18 months. As shown in Fig. 2 and Table 3, the efficacy of the molecular response at 1 month 
Table 2. Number of patients at baseline and responses of patients to dasatinib after 12 and 18 months of treatment

\begin{tabular}{ccccccc}
\hline \multirow{2}{*}{ Group $\begin{array}{c}\text { Ratio of copy number of } \\
B C R / A B L \text { mRNA to } A B L \\
\text { mRNA at baseline }\end{array}$} & \multicolumn{2}{c}{$\begin{array}{c}\text { Percentage of patients who } \\
\text { obtained an MMR, \%* }\end{array}$} & \multicolumn{2}{c}{$\begin{array}{c}\text { Percentage of patients who } \\
\text { obtained a CMR, \%** }\end{array}$} \\
\cline { 3 - 6 } & At 12 months & At 18 months & At 12 months & At 18 months \\
\hline A & $10 \%$ or more & $43.8(7 / 16)$ & $50(8 / 16)$ & 25 & $(4 / 16)$ & 25 \\
B & $1-10 \%$ & $92.3(12 / 13)$ & $92.3(12 / 13)$ & $38.5(5 / 13)$ & $38.5(5 / 13)$ \\
C & $0.1-1 \%$ & $76.2(16 / 21)$ & $85.7(18 / 21)$ & $33.3(7 / 21)$ & $47.6(10 / 21)$ \\
D & $0.1 \%$ or less (MMR) & NE & NE & NE & NE \\
\hline
\end{tabular}

*, number of patients who obtained an MMR/number of patients at baseline; **, number of patients who obtained an CMR/number of patients at baseline; CMR, complete molecular remission; MMR, major molecular remission; NE, not evaluable

Table 3. Major molecular remission and complete molecular remission achievement rates in four groups at one month after the start of dasatinib therapy

\begin{tabular}{|c|c|c|c|c|c|}
\hline \multirow{2}{*}{ Group } & \multirow{2}{*}{$\begin{array}{l}\text { Ratio of the copy number of } \\
B C R / A B L \text { mRNA for } A B L \\
\text { mRNA at } 1 \text { month after the } \\
\text { start of dasatinib }\end{array}$} & \multicolumn{2}{|c|}{$\begin{array}{l}\text { Percentage of patients who } \\
\text { obtained an MMR } \% *\end{array}$} & \multicolumn{2}{|c|}{$\begin{array}{l}\text { Percentage of patients who } \\
\text { obtained a CMR } \% * *\end{array}$} \\
\hline & & At 12 months & At 18 months & At 12 months & At 18 months \\
\hline $\mathrm{A}$ & $10 \%$ or more & $22.2(2 / 9)$ & $22.2(2 / 9)$ & $(0 / 9)$ & $(0 / 9)$ \\
\hline B & $1-10 \%$ & $80 \quad(8 / 10)$ & $80 \quad(8 / 10)$ & $(5 / 10)$ & $50 \quad(5 / 10)$ \\
\hline $\mathrm{C}$ & $0.1-1 \%$ & $77.8(14 / 18)$ & $77.8(14 / 18)$ & $33.3(6 / 18)$ & $44.4(8 / 18)$ \\
\hline $\mathrm{D}$ & $0.1 \%$ or less (MMR) & $100 \quad(11 / 11)$ & $100 \quad(11 / 11)$ & $45.5(5 / 11)$ & $54.5(6 / 11)$ \\
\hline
\end{tabular}

*, number of patients who obtained an MMR/number of patients at baseline; **, number of patients who obtained an CMR/number of patients at baseline; CMR, complete molecular remission; MMR, major molecular remission; NE, not evaluable

Table 4. Major molecular remission or complete molecular remission achievement rates in four groups at three months after the start of dasatinib therapy

\begin{tabular}{|c|c|c|c|c|c|}
\hline \multirow{2}{*}{ Group } & \multirow{2}{*}{$\begin{array}{l}\text { Ratio of copy number of } \\
B C R / A B L \text { mRNA to } A B L \\
\text { mRNA at } 3 \text { months after the } \\
\text { start of dasatinib }\end{array}$} & \multicolumn{2}{|c|}{$\begin{array}{c}\text { Percentage of patients who } \\
\text { obtained an MMR, \%* }\end{array}$} & \multicolumn{2}{|c|}{$\begin{array}{l}\text { Percentage of patients who } \\
\text { obtained a CMR, \%** }\end{array}$} \\
\hline & & At 12 months & At 18 months & At 12 months & At 18 months \\
\hline A & $10 \%$ or more & $0 \quad(0 / 4)$ & $0 \quad(0 / 4)$ & $0 \quad(0 / 4)$ & $0 \quad(0 / 4)$ \\
\hline B & $1-10 \%$ & $25 \quad(1 / 4)$ & $50 \quad(2 / 4)$ & $0 \quad(0 / 4)$ & $0 \quad(0 / 4)$ \\
\hline $\mathrm{C}$ & $0.1-1 \%$ & $66.7(10 / 15)$ & $73.3(11 / 15)$ & $13.3(2 / 15)$ & $44.4(8 / 18)$ \\
\hline $\mathrm{D}$ & $0.1 \%$ or less (MMR) & $100 \quad(21 / 21)$ & $100 \quad(21 / 21)$ & $57.1(12 / 21)$ & $66.7(14 / 21)$ \\
\hline
\end{tabular}

*, number of patients who obtained an MMR/number of patients number at baseline; **, number of patients who obtained an CMR/number of patients at baseline; CMR, complete molecular remission; MMR, major molecular remission; NE, not evaluable

after the initiation of dasatinib treatment was capable of predicting a significantly improved response consisting of the achievement of an MMR at 12 months $(p<0.003)$ or at 18 months $(p<0.003)$ and the achievement of a CMR at 18 months $(p<0.027)$. The efficacy of the molecular response at 3 months after the initiation of dasatinib treatment was also capable of predicting an improved response consisting of the achievement of an MMR at 12 months $(p<0.001)$ or an MMR at 18 months $(p<0.001)$ and the achievement of a CMR at 12 months $(p<0.002)$ or a CMR at 18 months $(p<$ 0.001) (Table 4).

\section{Response according to BCR-ABL mutation status}

Eight types of $B C R-A B L$ mutation were detected at baseline in $6(12 \%)$ of the $50 \mathrm{CML}-\mathrm{CP}$ patients. Double mutations were detected in two patients (No. 11: M244V and Q252H, and No. 47: P359V and F359I). Four patients (No. 24: A397P, No. 36: E459K, No. 47: P359V and F359I, and No. 62: M351T) had achieved a CMR at 12 months. One of the double-positive patients (No. 11) was unable to achieve an MMR or a CCyR, whereas the other double-positive patient (No. 47) was able to achieve a CMR at 12 months, as described above. Patient No. 15, who had an H396R mutation, was unable to achieve an MMR or a CCyR. A T315I 

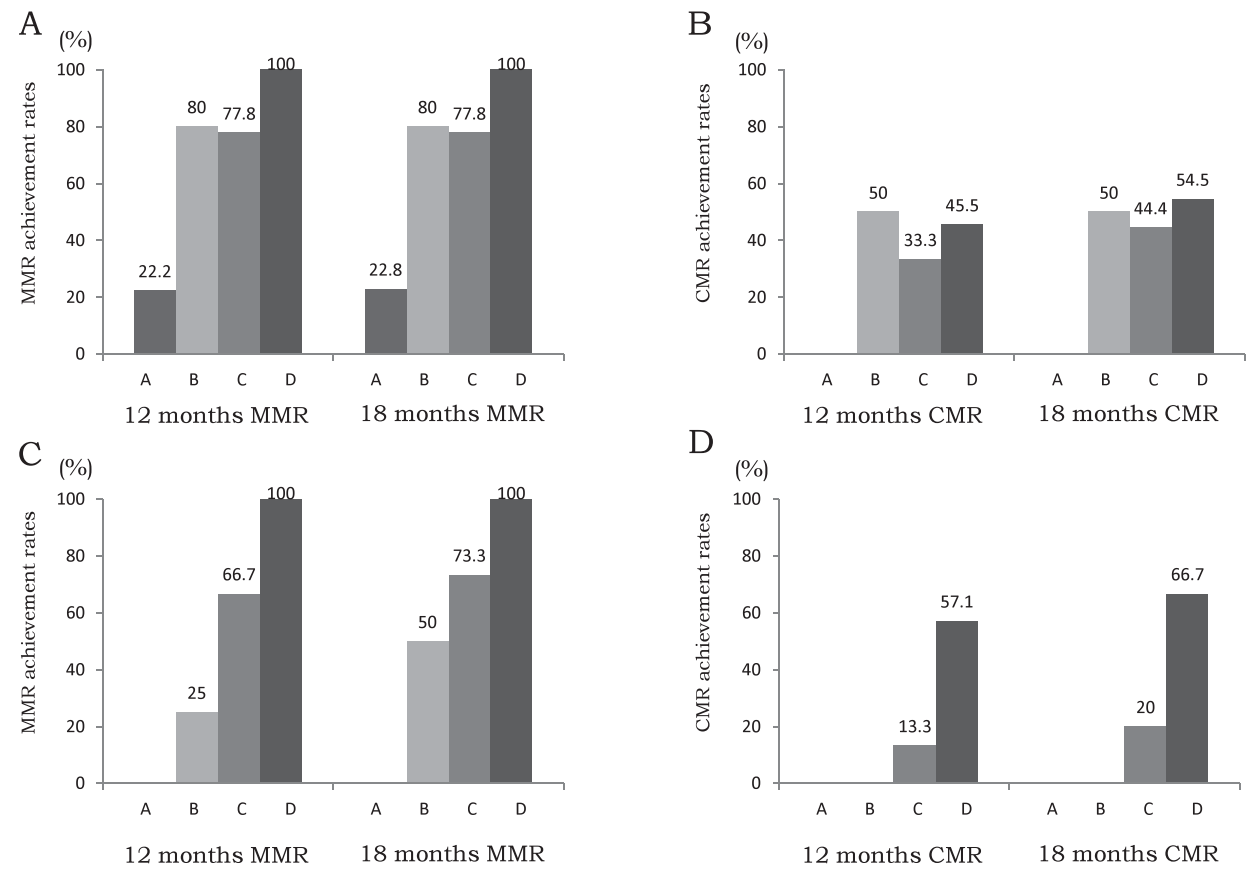

Fig. 2. Major molecular response (MMR) and complete molecular response (CMR) achievement rates in four groups at 1 and 3 months after the start of dasatinib therapy. (2A) MMR and (2B) CMR achievement rates in four groups at 1 month after the start of dasatinib therapy. (2C) MMR and (2D) CMR achievement rates in four groups at 3 months after the start of dasatinib therapy. The numbers on the bar graphs show the rates. $2 A, 2 B, 2 C$, and $2 D$ are the four patient subgroups (Table 2) divided according to the therapeutic effect of dasatinib.

mutation was identified in two patients (No. 25 \& 42) at three months. Both patients achieved a CCyR at 12 months, since hematopoietic stem cell transplantation was performed in both patients. A total of 14 different $B C R-A B L$ mutations affecting 12 different amino acids were detected.

\section{Safety}

Dasatinib was generally well tolerated, with 3 patients (5\%) having to discontinue treatment as a result of adverse hematological events (thrombocytopenia, anemia, neutropenia) at the 12-month follow-up.

Grade 1-2 cytopenia was a common hematologic adverse event, but was generally reversible and was effectively managed using dose adjustments (reductions or temporary interruptions). Six patients with grade 3-4 thrombocytopenia and three patients with grade 3-4 anemia were observed. However, no packed cells or platelet transfusions were administered in the present cohort.

Non-hematologic events that were considered by the investigator to be related to dasatinib therapy were generally mild to moderate in intensity (grade 1 or 2), with rashes, gastrointestinal disorders (diarrhea, nausea), and mild fever being occasionally observed. Dose reductions or interruptions were used effectively to treat the causes of elevated liver enzyme activities, headaches, bone pain, rashes, renal failure, cardiac abnormalities, and diarrhea. Twelve patients (24\%) experienced grade 1 or 2 pleural effusion; in general, however, most cases of pleural effusion were uncomplicated and were resolved with temporary dose interruptions, the administration of diuretics, or, in some cases, the administration of steroids.

The majority of the serum chemistry changes observed with dasatinib were also mild to moderate in intensity (grade 1 or 2); grade 3 to 4 aspartate aminotransferase elevation was observed in 2 patients (4\%), and 1 patient (2\%) experienced grade 3 to 4 alanine transaminase elevation.

\section{DISCUSSION}

This clinical trial examined imatinib-resistant and imatinib-intolerant patients with CML-CP who were treated with dasatinib, a second-generation BCR-ABL inhibitor.

Imatinib, the first BCR-ABL TKI approved for the treatment of Ph-positive CML and Ph-positive acute lymphocytic leukemia, has been shown to be clinically effective. However, resistance develops in some patients, and treatment options for patients who are resistant or intolerant to imatinib have been very limited. Dasatinib is a more potent inhibitor of the BCR-ABL protein tyrosine kinase. ${ }^{15,16}$ 
In a recent single-institution study of 119 imatinibresistant patients receiving dasatinib, nilotinib, or bosutinib as a second-line therapy for CML-CP, Milojkovic et al. ${ }^{25}$ found that patients achieving a $B C R-A B L \leq 10 \%$ at 3 months had significantly improved rates of progression-free survival, overall survival, CCyR, MMR, and CMR. Shah et al. ${ }^{26}$ supported the value of early molecular and cytogenetic responses in predicting the outcome of patients treated with second-line dasatinib therapy after imatinib failure.

Dasatinib (100 mg) administered once daily appears to offer a favorable long-term risk-benefit profile in patients with imatinib-resistant or imatinib-intolerant CML-CP. The present findings indicate that a consistent subgroup of CML$\mathrm{CP}$ patients who were resistant or intolerant to imatinib were able to obtain a molecular benefit from dasatinib therapy. We previously showed that a $B C R-A B L$ level had a significantly negative correlation with a relative increase in lymphocyte count at 1 and 3 months. ${ }^{21}$ Here, we were able to show that molecular and cytogenetic responses at 1 and 3 months were highly predictive of the outcomes at 12 and 18 months, and that the achievement of a $B C R-A B L \leq 0.1 \%$ (MMR) at 3 months was a particularly strong predictor. A lower level of $B C R-A B L$ transcript at 1 or 3 months was more strongly correlated with the $B C R-A B L$ transcript level at 12 and 18 months $(p<0.001)$ than a higher level of $B C R-A B L$. In particular, those with faster and deeper responses to dasatinib $(B C R-A B L \leq 10 \%$ at 1 month and $B C R-A B L \leq 1 \%$ at 3 months) were more likely to have a significantly improved MMR, and those with a $B C R-A B L$ level of $\leq 1 \%$ at 3 months were significantly more likely to have a CMR at 12 or 18 months $(p<0.001$ or $p<0.001)$.

These results are consistent with the findings reported by Hanfstein et al. ${ }^{27}$ showing that a group with a $1 \%$ IS at 3 months had the best CCyR and MMR, compared with a group with a $1 \%-10 \%$ IS or a group with a $10 \%$ IS. Hanfstein et al. proposed that a cut-off of a 1\% IS at 3 months seemed to be the best response-predictive surrogate, regardless of the treatment line (i.e., 1st line or $2 \mathrm{nd} / 3 \mathrm{rd}$ line) and TKI drug (i.e., imatinib vs. dasatinib vs. nilotinib). Since the transcriptional level of $B C R-A B L$ had a significantly negative correlation with the relative increase in lymphocyte count at 1 and 3 months, ${ }^{21}$ we speculated that the increase in the number of lymphocytes due to dasatinib therapy would be associated with improved responses to dasatinib, and that the lymphocytes themselves could inhibit the proliferation of leukemic cells through an immune-mediated effect. We here surmised that dasatinib may have a synergistic effect of direct TKI and anti-leukemic immunity.

The results of the present study show a high level of clinical activity for dasatinib in Japanese patients with CML. The rates of MMR and CMR were higher than overseas data. ${ }^{28}$ Overall, imatinib resistance or intolerance and the baseline $B C R-A B L$ mutation status did not appear to have an impact on the response to dasatinib for CML-CP. ${ }^{29}$

The most common drug-related adverse events were hematological suppression. Although there were side effects, such as rash, headache, nausea, vomiting, and pyrexia, grade 3 or 4 events were uncommon. The reduction or discontinuation of dasatinib prevented the appearance of grade 3 or 4 peripheral edema or pleural effusion when grade 1 or 2 side effects appeared. Although neutropenia and thrombocytopenia occurred in $43 \%$ and $49 \%$ of the patients, respectively, these events were generally manageable with dose discontinuation and/or reduction. In the present study, none of the patients required occasional support with hematopoietic growth factors or transfusions. Grade 3 or 4 hemorrhage from the gastrointestinal tract was not reported. The majority of serum biochemistry abnormalities that were observed were mild to moderate in severity. Although imatinib intolerance was present in 38\% (19/50) of the CML-CP patients, none of the patients experienced serious side effects or any side effects that led to the discontinuation of dasatinib administration.

In conclusion, although the number of Japanese patients that could be analyzed was relatively small, dasatinib exhibited a favorable long-term risk-benefit profile in patients with imatinib-resistant or imatinib-intolerant CML-CP. The present study is the first to show that a molecular response at 1 month was a strong predictor of outcome among patients with imatinib-resistant or imatinib-intolerant CML-CP.

\section{ACKNOWLEDGEMENTS}

This study was supported by the Epidemiological and Clinical Research Information Network (ECRIN). We thank Yumi Miyashita at ECRIN for collecting the data, and Yoshinori Yamamoto at BML for measuring the data.

\section{REFERENCES}

1 Wong S, Witte ON: The BCR-ABL story: bench to bedside and back. Annu Rev Immunol 22:247-306, 2004

2 Druker BJ, Sawyers CL, Kantarjian H, Resta DJ, Reese SF, et al.: Activity of a specific inhibitor of the BCR-ABL tyrosine kinase in the blast crisis of chronic myeloid leukemia and acute lymphoblastic leukemia with the Philadelphia chromosome. N Engl J Med 344:1038-1042, 2001

3 Druker BJ, Talpaz M, Resta DJ, Peng B, Buchdunger E, et al.: Efficacy and safety of a specific inhibitor of the BCR-ABL tyrosine kinase in chronic myeloid leukemia. N Engl J Med 344:10311037, 2001

4 Kantarjian HM, O'Brien S, Cortes JE, Smith TL, Rios MB, et al.: Treatment of Philadelphia chromosome-positive, acceleratedphase chronic myelogenous leukemia with imatinib mesylate. Clin Cancer Res 8:2167-2176, 2002

5 Druker BJ, Guilhot F, O'Brien SG, Gathmann I, Kantarjian H, et al.: Five-year follow-up of patients receiving imatinib for chronic 
myeloid leukemia. N Engl J Med 355:2408-2417, 2006

6 Gorre ME, Mohammed M, Ellwood K, Hsu N, Paquette R, et al.: Clinical resistance to STI-571 cancer therapy caused by BCRABL gene mutation or amplification. Science 293:876-880, 2001

7 Shah NP, Nicoll JM, Nagar B, Gorre ME, Paquette RL, et al.: Multiple BCR-ABL kinase domain mutations confer polyclonal resistance to the tyrosine kinase inhibitor imatinib (STI571) in chronic phase and blast crisis chronic myeloid leukemia. Cancer Cell 2:117-125, 2002

8 Hochhaus A, Hughes T: Clinical resistance to imatinib: mechanisms and implications. Hematol Oncol Clin North Am 18:641656,2004

9 Hochhaus A, Kreil S, Corbin AS, La Rosée P, Müller MC, et al.: Molecular and chromosomal mechanisms of resistance to imatinib (STI571) therapy. Leukemia.16:2190-2196, 2002

10 Illmer T, Schaich M, Platzbecker U, Freiberg-Richter J, Oelschlägel $\mathrm{U}$, et al:: P-glycoprotein-mediated drug efflux is a resistance mechanism of chronic myelogenous leukemia cells to treatment with imatinib mesylate. Leukemia 18:401-408, 2004

11 Thomas J, Wang L, Clark RE, Pirmohamed M: Active transport of imatinib into and out of cells: implications for drug resistance. Blood 104:3739-3745, 2004

12 Dai Y, Rahmani M, Corey SJ, Dent P, Grant SA: A Bcr/Ablindependent, Lyn-dependent form of imatinib mesylate (STI-571) resistance is associated with altered expression of Bcl-2. J Biol Chem 279:34227-34239, 2004

13 Donato NJ, Wu JY, Stapley J, Gallick G, Lin H, et al.: BCR$\mathrm{ABL}$ independence and LYN kinase overexpression in chronic myelogenous leukemia cells selected for resistance to STI571. Blood 101:690-698, 2003

14 O'Hare T, Walters DK, Stoffregen EP, Jia T, Manley PW, et al:: In vitro activity of Bcr-Abl inhibitors AMN107 and BMS-354825 against clinically relevant imatinib-resistant Abl kinase domain mutants. Cancer Res 65:4500-4505, 2005

15 Kantarjian H, Pasquini R, Hamerschlak N, Rousselot P, Holowiecki J, et al.: Dasatinib or high-dose imatinib for chronicphase chronic myeloid leukemia after failure of first-line imatinib: a randomized phase 2 trial. Blood 109:5143-5150, 2007

16 Hochhaus A, Kantarjian HM, Baccarani M, Lipton JH, Apperley $\mathrm{JF}$, et al.: Dasatinib induces notable hematologic and cytogenetic responses in chronic-phase chronic myeloid leukemia after failure of imatinib therapy. Blood 109:2303-2309, 2007

17 Guilhot F, Apperley J, Kim DW, Bullorsky EO, Baccarani M, et al.: Dasatinib induces significant hematologic and cytogenetic responses in patients with imatinib-resistant or -intolerant chronic myeloid leukemia in accelerated phase. Blood 109:4143-4150, 2007

18 Cortes J, Rousselot P, Kim DW, Ritchie E, Hamerschlak N, et al.: Dasatinib induces complete hematologic and cytogenetic responses in patients with imatinib-resistant or -intolerant chronic myeloid leukemia in blast crisis. Blood 109:3207-3213, 2007

19 Ottmann O, Dombret H, Martinelli G, Simonsson B, Guilhot F, et al.: Dasatinib induces rapid hematologic and cytogenetic re- sponses in adult patients with Philadelphia chromosome positive acute lymphoblastic leukemia with resistance or intolerance to imatinib: interim results of a phase 2 study. Blood 110:23092315, 2007

20 Mauro MJ, Baccarani M, Cervantes F, Lipton JH, Matloub R, et al.: Dasatinib 2-year efficacy in patients with chronic-phase chronic myelogenous leukemia (CML-CP) with resistance or intolerance to imatinib (START-C). J Clin Oncol 26 (Suppl 18): 7009a, 2008

21 Yoshida C, Fletcher L, Ohashi K, Wakita H, Kumagai T, et al.: Harmonization of molecular monitoring of chronic myeloid leukemia therapy in Japan. Int J Clin Oncol 17:584-589, 2012

22 Kumagai T, Matsuki E, Inokuchi K, Ohashi K, Shinagawa A, et al.: Relative increase in lymphocytes from as early as 1 month predicts improved response to dasatinib in chronic-phase chronic myelogenous leukemia. Int J Hematol 99:41-52, 2014

23 Inokuchi K, Shinohara T, Futaki M, Hanawa H, Tanosaki S, et al.: Establishment of a cell line with variant BCR/ABL breakpoint expressing P180BCR/ABL from late-appearing Philadelphiapositive acute biphenotypic leukemia. Genes Chromosomes Cancer 23:227-238, 1998

24 Inami M, Inokuchi K, Nakayama K, Tamura H, Shimada $\mathrm{T}$, et al.: Simultaneous novel BCR-ABL gene mutation and increased expression of BCR-ABL mRNA caused clinical resistance to STI571 in double-Ph-positive acute biphenotypic leukemia. Int J Hematol 78:173-175, 2003

25 Müller MC, Cortes JE, Kim DW, Druker BJ, Erben P, et al.: Dasatinib treatment of chronic-phase chronic myeloid leukemia: analysis of responses according to preexisting BCR-ABL mutations. Blood 114:4944-4953, 2009

26 Milojkovic D, Apperley JF, Gerrard G, Ibrahim AR, Szydlo R, et al:: Responses to second-line tyrosine kinase inhibitors are durable: an intention-to-treat analysis in chronic myeloid leukemia patients. Blood 119:1838-1843, 2012

27 Shah NP, Guilhot F, Cortes JE, Schiffer CA, Le Coutre P, et al.: Long-term outcome with dasatinib after imatinib failure in chronic-phase chronic myeloid leukemia: follow-up of phase 3 study. Blood 123:2317-2324, 2014

28 Hanfstein B, Müller MC, Hehlmann R, Erben P, Lauseker M, et al.: Early molecular and cytogenetic response is predictive for long-term progression-free and overall survival in chronic myeloid leukemia (CML). Leukemia 26:2096-2102, 2012

29 Fujisawa S, Nakamae H, Ogura M, Ishizawa K, Taniwaki M, et al.: Efficacy and safety of dasatinib versus imatinib in Japanese patients with newly diagnosed chronic-phase chronic myeloid leukemia (CML-CP): Subset analysis of the DASISION trial with 2year follow-up. Int J Hematol 99:141-153, 2014

30 Shah NP, Kim DW, Kantarjian H, Rousselot P, Llacer PED, et al.: Potent, transient inhibition of BCR-ABL with dasatinib $100 \mathrm{mg}$ daily achieves rapid and durable cytogenetic responses and high transformation-free survival rates in chronic phase chronic myeloid leukemia patients with resistance, suboptimal response or intolerance to imatinib. Haematologica 95:232-240, 2010 